\title{
Migración latinoamericana y caribeña, trayectorias laborales y precariedad laboral en la Ciudad de Temuco*
}

\author{
Latin American and Caribbean migration, employment trajectories \\ and job insecurity in the City of Temuco
}

\author{
Ana Huento Nahuelan*
}

Resumen: La presente investigación se desarrolla bajo el enfoque socio-critico desde la perspectiva de la Investigación-Acción de Fals Borda. Este propone el trabajo con los grupos minoritarios de las sociedades, quienes son explotados en múltiples dimensiones de la vida social y laboral, considerando para efectos de esta investigación. El objetivo general de nuestra labor fue conocer las trayectorias de trabajadores migrantes latinoamericanos y del Caribe en la ciudad de Temuco. Con relación a ello, se desprenden los siguientes objetivos específicos: 1) Analizar las dimensiones de la precariedad de las personas migrantes latinoamericanas y del caribe en la ciudad de Temuco, 2) Identificar la ocupación laboral de los migrantes provenientes de américa latina y el caribe en su país de origen y en el país de destino y 3) Abrir espacios de discusión y devolución de contenido con las diferentes organizaciones y colectivos, en torno a las condiciones de precariedad laboral.

Palabras clave: migración, empleo, pobreza, impacto social, organización social

\begin{abstract}
This research is developed under the socio-critical approach from the perspective of Action Research of Fals Borda. This proposes work with minority groups in societies, who are exploited in multiple dimensions of social and work life, considering for the purposes of this research. The general objective of our work was to know the trajectories of Latin American and Caribbean migrant workers in the city of Temuco. In relation to this, the following specific objectives emerge: 1) Analyze the dimensions of the precariousness of Latin American and Caribbean migrants in the city of Temuco, 2) Identify the occupational occupation of migrants from Latin America and the Caribbean in their country of origin and in the country of destination and 3) Open spaces for discussion and return of content with different organizations and groups, regarding precarious employment conditions.
\end{abstract}

Keywords: migration, employment, poverty, social impact, social organization

Recibido: 23 mayo 2020 Aceptado: 4 agosto 2020

\footnotetext{
* Esta investigación fue parte del proyecto FONDECYT Regular No. 1161347, titulado: "Cartografía de la(s) precariedad(es) laboral(es) y las relaciones laborales de la zona centro sur. Tipología del trabajo precario y su incidencia en la práctica sindical en las regiones del Maule, Biobío y la Araucanía".

* Trabajadora Social, Universidad Católica de Temuco, anafremanente2010@gmail.com
} 


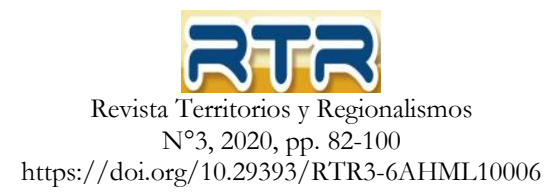

\section{Introducción}

Las migraciones laborales constituyen un foco de estudio importante ya que significan un desafío para sociedades que se encuentran en desarrollo, debido al aumento de diferencias entre países con un mayor desarrollo económico, las cuales garantizarían mayores y mejores oportunidades de empleo. La geografía a través de Ravenstein ${ }^{1}$, hizo los primeros aportes en temas migratorios, publicando leyes bajo las cuales se explicaría el proceso migratorio, donde el factor económico se consideraba como la causa más importante para la entrada y salida de personas en búsqueda de nuevas y mejores oportunidades de trabajo que les permitiesen cubrir las carencias. Este proceso hablaba de la creación de una dependencia entre comercio-desarrollo y oferta-demanda.

La teoría de la Dependencia propuesta por Cardoso y $\mathrm{Faleto}^{2}$, hace distinciones entre dos grandes conceptos, "Centro", refiriéndose a las sociedades completamente desarrolladas cuya característica principal era disponer mayor cantidad de empleo, y "Periferia", lugar desde donde se desplazaban trabajadores para ocupar los puestos vacantes. Mabogunje ${ }^{3}$ plantea que, más que ver a los individuos como personas con motivos propios, la migración se trata de estados-naciones que realizan proyectos para sus ciudadanos que los obligan a salir de ellas por diversas razones, entre las que destacan crisis políticas, faltas de empleo, desvalorización del trabajo, etc.

En el Enfoque Transnacional según García 4 , la importancia de ver al trabajador migrante como un sujeto social recae en las transformaciones asociadas a la forma de entender la migración, es decir, contraponer lo que se deja versus lo que se recibe en el lugar de destino, tanto por parte de la economía como las implicancias sociales de apoyo, contención y desarrollo de actividades laborales que les garanticen seguridad en todas las dimensiones que inciden en su bienestar.

Chávez y Preciados ${ }^{5}$ añaden que este enfoque permite un análisis más profundo sobre cómo el trabajador/a migrante compatibiliza su rol laboral y socioafectivo, pues esta perspectiva comprende y reconoce los vínculos que mantiene el/la migrante con su familia en origen o lugar de procedencia. Sin embargo, Waldinger ${ }^{6}$, plantea que existe el riesgo de que el migrante se separe de sus roles socioafectivos debido a que el insertarse en la sociedad de destino ofrece relaciones sociales más amplias y de fácil acceso.

El transnacionalismo, de acuerdo con Parella y Cavalcanti ${ }^{7}$, permite estudiar las trayectorias migratorias entendidas como desplazamientos de personas desde su lugar de procedencia hacia un destino determinado, estos desplazamientos se comprenden de acuerdo con los lugares en los que ha estado residiendo el migrante (tanto dentro como fuera de su país de origen). Moret ${ }^{8}$ plantea que las trayectorias laborales o las experiencias que ha tenido la persona migrante en el mundo del trabajo, le garantizaría mayor o menor seguridad laboral, ya que, de acuerdo a su versatilidad, los

${ }^{1}$ Ernst Ravenstein, “The laws of migration”, Journal of the statistical society of London, 48(2), pp. 167-235, 1885

2 Fernando Cardoso \& Enzo Faletto, Dependencia y desarrollo en América Latina: ensayo de interpretación sociológica, (No. 330.13/C26d), Siglo Veintiuno, 1971

3 Akin Mabogunje, "Systems Approach to a Theory of Rural-Urban Migration", Geographical Analysis, v. 2, n. 1, p. 1-18, 1970

${ }^{4}$ Francisco Pinilla, La degradación de las condiciones de trabajo: Tendencias de flexibilización en las relaciones de empleo, intensificación del esfuerzo y consecuencias en la salud de los trabajadores, Doctoral dissertation, Universidad Nacional de Educación a Distancia., UNED, 2015

5 Guillermina Chávez \& Susana Preciados, "Comunidad transnacional e interacción comunicativa: diagnóstico social" PROSPECTIVA. Revista de Trabajo Social e Intervención Social, pp.37-62, 2018

${ }^{6}$ Roger Waldinger, "Más allá del transnacionalismo: Una perspectiva alternativa de la conexión de los inmigrantes con su país de origen", Migraciones Internacionales, 7(1), pp. 189-220. https://doi.org/10.17428/rmi.v7i1.690, 2017

${ }^{7}$ Sonia Parella \& Leonardo Cavalcanti, "Aplicación de los campos sociales transnacionales en los estudios sobre migraciones", en C. Solé, S. Parella, L. Cavalcanti (Coords.), Nuevos retos del transnacionalismo en el estudio de las migraciones, Ministerio de Trabajo e Inmigración, Subdirección General de Información Administrativa y Publicaciones, Madrid, 2009

8 Joelle Moret, "Cross-border mobility, transnationality and ethnicity as resources: european Somalis' post-migration mobility practices”, Journal of Ethnic and Migration Studies, 42(9), pp. 1455-1472, 2016 


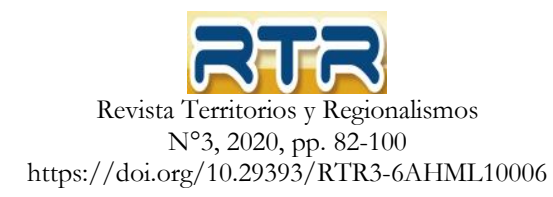

trabajadores/as que han estado en más de un empleo cumpliendo diversas funciones se encuentran mejor preparados para enfrentarse al mundo laboral en el país de destino.

Los trabajadores migrantes se insertan en Chile principalmente en el área de la construcción (hombres) y servicio doméstico (mujeres), ya que son las áreas que requieren mayor número de trabajadores, constituyéndose como una puerta de entrada para insertarse laboralmente, debido a que no se requiere una alta cualificación para obtener empleos en estos rubros, y considerando que la validación de títulos profesionales sigue siendo otra de las dificultades que enfrenta la población migrante?

En estas prácticas de inserción laboral, a la vez, se presenta una triple discriminación por sexo, raza y clase, ejercida por parte de sus empleadores. Esta discriminación, como estructura de la violencia, es constatable en que las trabajadoras migrantes son vistas y representadas como personas que cumplen funciones predeterminadas por construcciones de roles de género (como el cuidado y quehaceres propios de hogar), o por una hipersexualización de los cuerpos. Por tanto, se genera una estigmatización y se crean imaginarios sociales sobre la historia de vida de las trabajadoras ${ }^{10}$.

El trabajo representa desigualdad, exclusión y precarización hacia trabajadores/as migrantes, ya que según Stefoni, Leiva y Bonhomme ${ }^{11}$, este grupo se inserta en condiciones laborales mínimas, debido a que ello constituye su puerta de entrada a establecerse laboralmente en el país de destino, ocupando los "nichos laborales" o puestos de empleos descartados por los trabajadores nacionales. Sin embargo, esto representa un riesgo multidimensional, pues los impulsa a vivenciar precariedad laboral, la cual según Julián ${ }^{12}$ se comprende como:

"Deterioro de las condiciones laborales, a una condición de inestabilidad laboral; un lugar en el espacio social donde el/la trabajador/a se encuentra desprotegido/a ante la expansión de las relaciones no formales, donde las leyes no lo protegen, la consolidación de un área de desprotección” (p.150).

En otras palabras, la formalidad o informalidad del trabajo desempeñado sería un elemento central a la hora de delimitar la existencia o inexistencia de deterioro en las condiciones laborales, lo que afectaría directamente en otros ámbitos de la vida del migrante. Julián ${ }^{13}$ hace una revisión de como incidiría esta conceptualización en la vida de este grupo de trabajadores/as, donde se deprenden dimensiones asociadas a lo político-social (vínculos de los trabajadores a organizaciones colectivas), cultural-epistemológica (centrada en la historicidad del trabajador como sujeto social) y la dimensión económica.

La transnacionalidad ${ }^{14}$ realiza un acercamiento aún más profundo de la importancia que adquiere la dimensión socio-familiar para los trabajadores/as migrantes, dado que el proceso migratorio se piensa en función de sus repercusiones en el ámbito familiar, ya que, de acuerdo con Zapata ${ }^{15}$, las

\footnotetext{
9 Marcela Tapia Ladino, "Frontera, movilidad y circulación reciente de peruanos y bolivianos en el norte de chile", Estudios atacameños, (50), pp. 195-213. https://doi.org/10.4067/S0718-10432015000100010, 2015

${ }^{10}$ Sonia Parella, Mujer, inmigrante y trabajadora: La triple discriminación, Anthropos Editorial, Barcelona, 2003

11 Carolina Stefoni, Sandra Leiva \& Macarena Bonhomme, "Migración internacional y precariedad laboral. El caso de la industria de la construcción en Chile", Revista Interdisciplinar da Mobilidade Humana, 25(49). Recuperado de http://www.csem.org.br/remhu/index.php/remhu/article/view/717, 2017

${ }^{12}$ Dasten Julián, "La precariedad laboral, modernidad y modernización capitalista: Una contribución al debate desde América Latina", Trabajo y sociedad, (23), pp. 147-168, 2014

13 Dasten Julián, "Precariedad laboral en América Latina: contribuciones a un modelo pata armar", Revista Colombiana de Sociología, 40(2), pp. 27-46, 2017

14 Parella \& Cavalcanti, op. cit.

15 Adriana Zapata, "Madres y padres en contextos transnacionales: el cuidado desde el género y la familia", Desacatos, (52), pp. $14-31,2016$
} 


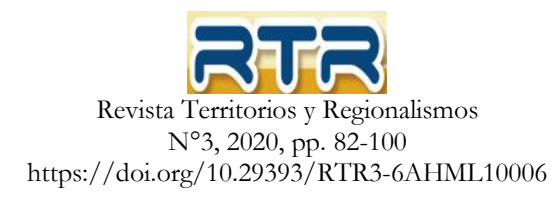

relaciones parentales son las razones más importantes a la hora de elegir el país de destino, ya que para cada uno de los trabajadores migrantes más que su desarrollo laboral, importa el bienestar, tranquilidad y seguridad de sus hijos y familia.

La organización social y sus prácticas vinculadas a la migración y el trabajo permiten la inserción de esta población en la sociedad de destino, provocando un cambio tanto en los propios migrantes como quienes reciben a este grupo, pues se generan lazos de cooperación y entendimiento que configuran nuevas formas de constituir una sociedad, donde es vital la escucha, la empatía y la orientación hacia una estabilidad psico social que le garantice al migrante que tanto él como su grupo familiar están resguardados de cualquier situación problema.

Las redes migratorias se constituyen a través de relaciones sociales entre migrantes, establecidas entre migrantes y no migrantes en el país de destino como lazos donde es posible encontrar el soporte necesario frente a cualquier dificultad. Gómez \& Torres ${ }^{16}$ plantean que este acompañamiento solo es durante el proceso de inserción, sin embargo, Portes, Guarnizo y Landot en Ávila ${ }^{17}$, añaden que las redes migratorias no sólo se activan de manera esporádica frente a situaciones eventuales, sino que también funcionan permanentemente como refuerzo de la identidad del migrante.

El sindicalismo nace de los cuestionamientos por parte de los trabajadores que se encontraban insertos en el mundo laboral mundial, principalmente latinoamericano en la década de los 70’ y 80', quienes vivieron la transición entre las negociaciones directas hacia las intermediarias, como lo señala Várguez:

"Los sindicatos de empresa se caracterizaron por negociar directamente con los patrones, relacionarse estrechamente con dirigentes y obreros, y no tener el apoyo de las organizaciones nacionales. En cambio, los sindicatos organizados en estas centrales se identificaron por negociar a través de sus dirigentes, tener poca o nula participación de los trabajadores en las negociaciones y por la relación muy cercana con las autoridades del Estado ${ }^{18 ”}$. (p.460).

Las organizaciones sindicales, representaban y representan aún en la actualidad una oportunidad para contrarrestar la precariedad laboral de los grupos de trabajadores más vulnerables, ya que propician espacios de ayuda, orientación y abogacía para los trabajadores adscritos, sin embargo, el sindicalismo a nivel latinoamericano aún se encuentra pasando por una etapa de reivindicación y reorganización, tras la época de persecuciones de dictaduras militares. Ello provocó una confusión en cuanto al rol y a los alcances de este frente al empresariado regulador de la economía y al Estado ${ }^{19}$. Así mismo Los principales desafíos de los Sindicatos, de acuerdo con Julián son

"Los objetivos principales delineados para con el sindicalismo son: a) alcanzar diálogos intersindicales de carácter horizontal y convertirse en interlocutor de los actores sociales; y b) proceder en acciones colectivas hacia demandas que se articulen desde el mundo del trabajo potenciando el rol sociopolítico del sindicalismo en la sociedad ${ }^{20}$ " (p.190).

\footnotetext{
16 Pablo Gómez \& Víctor Torres, "Redes sociales en la migración paraguaya y brasilera hacia la Argentina. Un análisis comparativo de las redes de ayuda y los vínculos binacionales", Anais, pp. 1-20, 2016

${ }^{17}$ Javier Ávila, "Repensando la etnicidad y el transnacionalismo desde el análisis de redes personales", Redes. Revista hispana para el análisis de redes sociales, 26(2), pp. 158-170, 2015

${ }^{18}$ Luis Várguez, "Francisco Zapata, El sindicalismo latinoamericano", Estudios sociológicos, 35(104), pp. 457-461, 2017

19 Arturo Martínez, "Estrategia Sindical en un Chile Internacionalizado", en Jaime Ensignia (editor), Mitos y realidades del mercado laboral en Chile, pp.199-212, Friedrich Ebert Stiftung, Santiago, 2005

${ }^{20}$ Dasten Julián, "Identidades y re-significados del actor sindical. Diez Núcleos de tensión del sindicalismo en Chile", Andamios, 13(30), pp. 171-194, 2016
} 


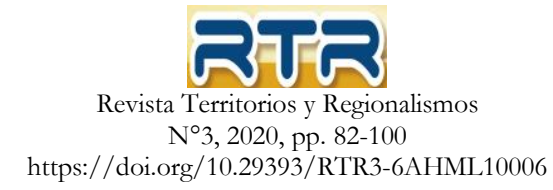

Es decir, el sindicato tiene un trabajo muy importante por realizar y es el de retomar sus inicios y trabajar sobre premisas colectivas, estableciendo una educación al interior de sus miembros y dirigentes respecto a los cambios que el proceso de globalización ha generado a nivel latinoamericano, nacional y local, los cuales beneficiaron a las grandes empresas aumentando la producción, pero mantuvieron y, en la gran mayoría, disminuyeron los salarios de los trabajadores. En el caso puntual de Chile, los sindicatos debieron enfrentar el cambio del contrato directo con el empleador a ser contratados por empresas mediadoras o subcontratistas, por obras terminadas, aumentando la inseguridad e inestabilidad del sector terciario de servicios, especialmente en el rubro de la construcción

\section{Materiales y Métodos}

La presente investigación, se desarrolló bajo el enfoque socio-critico desde la perspectiva de la Investigación-Acción de Fals Borda ${ }^{21}$, quien propone el trabajo con los grupos minoritarios de las sociedades, quienes son explotados en múltiples dimensiones de la vida social y laboral, considerando para efectos de esta investigación. El objetivo general de este proceso fue conocer las trayectorias de trabajadores migrantes latinoamericanos y del Caribe, en relación a ello, se desprenden los siguientes objetivos específicos: 1) Analizar las dimensiones de la precariedad de las personas migrantes latinoamericanas y del caribe en la ciudad de Temuco, 2) Identificar la ocupación laboral de los migrantes provenientes de américa latina y el caribe en su país de origen y en el país de destino y 3) Abrir espacios de discusión y devolución de contenido con las diferentes organizaciones y colectivos, en torno a las condiciones de precariedad laboral.

El grupo de informantes clave estuvo compuesto por: trabajadores/as migrantes Latinoamericanos y del Caribe, siendo 3 migrantes de nacionalidad ecuatoriana, 2 de nacionalidad haitiana y 2 trabajadores/as de nacionalidad venezolana. Los criterios de selección fueron el tiempo de residencia en el país, el cual se fijó en un mínimo de 6 meses y que se encontrasen trabajando de manera formal y/o informal, considerando tanto trabajadores dependientes como independientes.

Las proporciones de género quedaron en 3 hombres y 4 mujeres. Cabe mencionar que para dar cumplimiento al tercer objetivo específico se realizó un conversatorio denominado Caminos de encuentro. Sindicalismo y Migración, donde trabajadores migrantes (sindicalizados y no sindicalizados), dirigentes sindicales y la comunidad en general, pudieron encontrarse y exponer sus puntos de vista respecto al mundo del trabajo chileno.

Las consideraciones éticas de esta investigación contemplan la declaración de Singapur de acuerdo lo establece la Comisión Nacional de Investigación Científica y Tecnológica22, por otra parte, se consideran los criterios de validez interna, credibilidad y transferibilidad ${ }^{23}$.

La técnica de recolección de datos fue la entrevista en profundidad que, según Taylor y Bogdan, se define como una técnica cuyo propósito es:

"Adentrase en la vida del otro, penetrar y detallar en lo trascendente, descifrar y comprender los gustos, los miedos, las satisfacciones, las angustias, zozobras y alegrías, significativas y relevantes del entrevistado; consiste en construir paso a paso y minuciosamente la experiencia del otro24" (p.101).

\footnotetext{
${ }^{21}$ Fals Borda, Una sociología sentipensante para América Latina, (No. 316 301.098). e-libro, Corp, 2009

22 Comisión Nacional de Investigación Científica y Tecnológica, "Declaración de Singapur sobre la integridad en la investigación", link de consulta:

https://www.conicyt.cl/fondap/files/2014/12/DECLARACI\%C3\%93N-SINGAPUR.pdf

${ }^{23}$ Yadira Corral, "Validez y Fiabilidad en Investigaciones Cualitativas", Revista Arjé, pp. 11-14, 2017

${ }^{24}$ Steven Taylor \& Robert Bogdan, Introducción a los métodos cualitativos de investigación, Paidós, Barcelona, 1990
} 


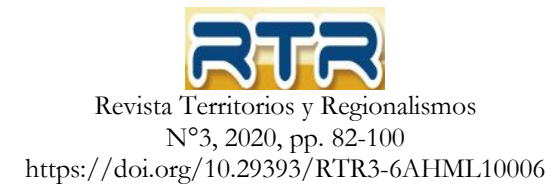

Es decir, se busca la deconstrucción lo más completa posible, desde un plano igualitario. $\mathrm{El}$ análisis fue de carácter cualitativo ${ }^{25}$, se centra en las vivencias de los sujetos para así dar cuenta de las distintas realidades que considera el proceso migratorio, se considera la utilización del software Atlas ti y un análisis lógico-semántico, en el que se plantea 4 aspectos a considerar:

“1) gramatical: estudia la analogía, la sintaxis, la prosodia y la ortografía de las palabras; 2) lógico: estudia la formación, naturaleza y sintaxis de las proposiciones; 3 ) literario: estudia la elocución, figuras retóricas, etc.; 4) lexigráfico: estudia la significación de las palabras y su etimología ${ }^{26 "}$ (p. 132).

En otras palabras, es una explicación exhaustiva y detallada sobre cada respuesta, sin embargo, para efectos de la complejidad y temporalidad en la que se suscitaron las entrevistas (octubrenoviembre, 2018), se hizo un análisis por cada dimensión a la que responde la pauta. Cabe destacar que paralelamente a este proceso de investigación se desarrolló un trabajo formativo y colaborativo con organizaciones migrantes de la ciudad, quienes fueron pilares importantes a la hora de vincular el mundo del trabajo y el proceso migratorio.

\section{Resultados}

A continuación, se presentan las categorías que arrojó el proceso de análisis de esta investigación, se deja claro que debido a la temática abordada no aparecieron todas las dimensiones contempladas en el instrumento (situación, sentido y narrativa de la precariedad, impactos en esferas sociales y ampliadas de la vida del trabajo, asociatividad, solidaridades y formas de acción colectiva relacionadas principalmente con el mundo sindical) debido a que los entrevistados no poseen conocimientos sobre sindicatos, se agregaron las siguientes categorías de análisis: Indefensión frente a Políticas Públicas, Nichos Laborales y redes. Además, se presenta una breve síntesis de conversatorio realizado en el marco de esta investigación denominado Caminos de encuentro: Sindicalismo y Migración.

\section{Situación, sentido y narrativa de la precariedad}

En la presente dimensión se buscó indagar sobre hitos relevantes acerca de la biografía del trabajo en el país de origen y destino. Para esta dimensión se consideraron cinco preguntas.

Figura 1: Esquema resumen de dimensión: situación, sentido y narrativa de la precariedad

${ }^{25}$ Roberto Hernández, Carlos Fernández \& Pilar Baptista, Metodología de la investigación (5a ed), McGraw-Hill, México, D.F, 2010

26 Alfonso Zamorano, "Análisis lógico y análisis gramatical en Colombia (s. XX): la teoría lingüística de Próspero González Ruiz”, Revista argentina de bistoriografía lingüistica, 2(2), pp. 125-143, 2016 


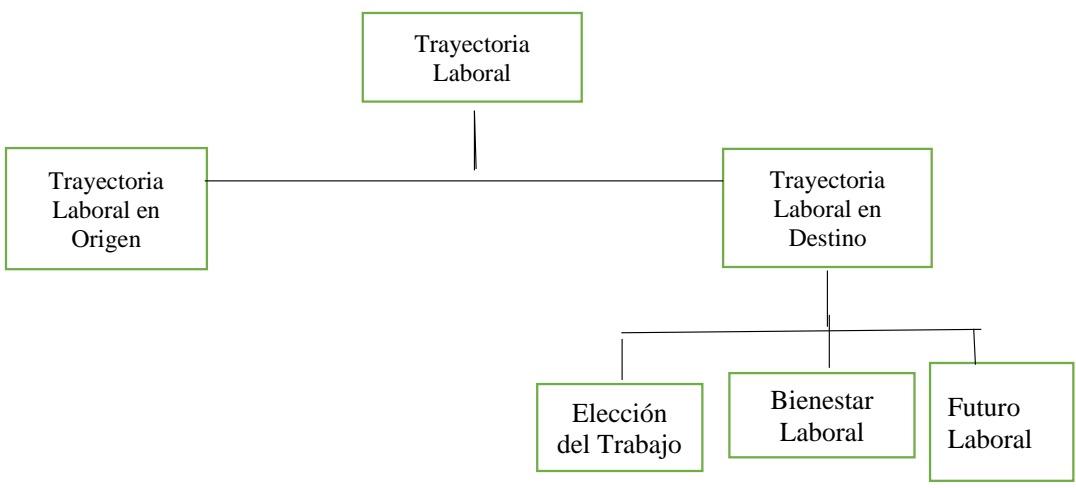

Trayectoria Laboral es la categoría principal la cual se refiere a la cantidad de trabajos que ha tenido desde sus inicios como trabajado/a:

"Desde muy pequeña comencé a trabajar, pequeña relativamente para uno que deja la infancia ya que a los 1718 años comienzas a trabajar, tenía un familiar con un contacto en una librería y fuera de colegio iba y la ayudaba en las tardes. A partir de los 18 independiente en ese sentido económico" (Trabajadora Dependiente 1).

Trayectoria laboral en origen constituye la primera categoría la cual guarda relación con los trabajos que el migrante ha tenido en su país de origen y los ámbitos en los que se ha desempeñado,

"A ver, he trabajado en costura, cuidando a niños, he trabajado en una panadería, eh he trabajado atendiendo un local y atendiendo una zapatería" (Trabajadora Independiente 2). en la vida del migrante.

De ella se desprende la subcategoría narrativa de vida apuntando a hechos significativos

"Este lo más terrible que me ha pasado al salir de allá fue que tenía 12 días en ecuador, muere mi papá y no pude regresar ni ir a enterrarlo, eso ha sido lo que más he lamentado yo" (Trabajadora Dependiente 1).

Trayectoria Laboral en destino es la segunda categoría, la cual se relaciona con los trabajos que realiza el migrante en la actualidad

"Bueno mi trabajo acá en Chile se basa en el comercio tanto en la artesanía o paquetería porque trabajo, más que todo en el verano trabajo en ferias costumbristas o religiosas que hacen en diferentes ciudades" (Trabajador Independiente 1)

Elección del trabajo es la segunda subcategoría y se relaciona con los motivos que los llevaron a elegir su trabajo, ante ello las trabajadoras migrantes dependientes señalan:

"Porque necesitaba trabajar, ya que no había traído mucho dinero y aparte tengo que pagar arriendo, comida, mandarle a mi familia y segundo porque me ofrecieron el 


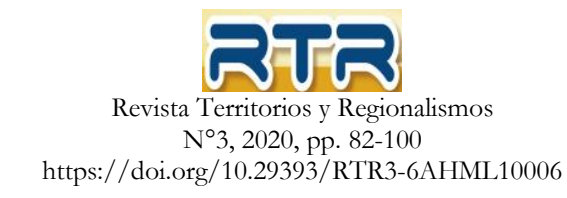

contrato para poder también agilizar lo de mi proceso migratorio y porque bueno me gusto el trabajo, y ya yo venía como con mente de que voy a trabajar de lo que consiga y como allí me lo consiguieron, y mi jefa ha sido de verdad muy buena conmigo me quede ahí" (Trabajadora Dependiente 2).

"Tenía que buscar donde vivir y me hablaron de esta posibilidad de puerta adentro, que significaba que podía trabajar y vivir en la misma casa donde yo trabajara por lo menos de lunes a viernes" (Trabajadora Dependiente 1).

Bienestar Laboral constituye la tercera subcategoría que guarda relación con la capacidad del trabajo de generar seguridad y tranquilidad en la persona haciendo énfasis en cómo ha sido su inserción laboral y si esta le permite vivir de acuerdo con lo esperado.

“A nivel de vida bien porque puedo pagar mi arriendo, puedo pagar mis gastos y lo más importante que puedo mandarle dinero a mi familia. Y estoy tranquila allí también, igual es temporal, entonces sí implica un beneficio para mi familia" (Trabajadora Dependiente 2).

Futuro Laboral como última subcategoría se relaciona con las proyecciones en materia laboral de los trabajadores/as migrantes.

"Tengo muchas, pero no, o sea eso es lo primer, me gustaría tener una casa, porque yo sé que en una casa me ahorraría tanto, el mensual del arriendo y con eso ya trataría de hacer cualquier cosa, de vender comida, porque yo he pensado de ponerme un taller, un taller de costura o vender comida, eso" (Trabajadora Independiente 1).

Los entrevistados se abren acerca de sus experiencias laborales y de vida personales en sus países de origen, siendo estas de carácter principalmente económico y político, haciendo alusión principalmente a que debido a una búsqueda de estabilidad para sus familias deciden emigrar considerando aún lo difícil que es conseguir un puesto de trabajo sin estudios y con estudios, pues deben adecuarse a la oferta que tiene para ellos el país de destino.

\section{Proceso de Trabajo}

Relaciona al migrante directamente con cómo se vincula con el mundo del trabajo en el país de destino, esta dimensión considera siete preguntas.

Figura 2: Esquema resumen dimensión proceso de trabajo. 


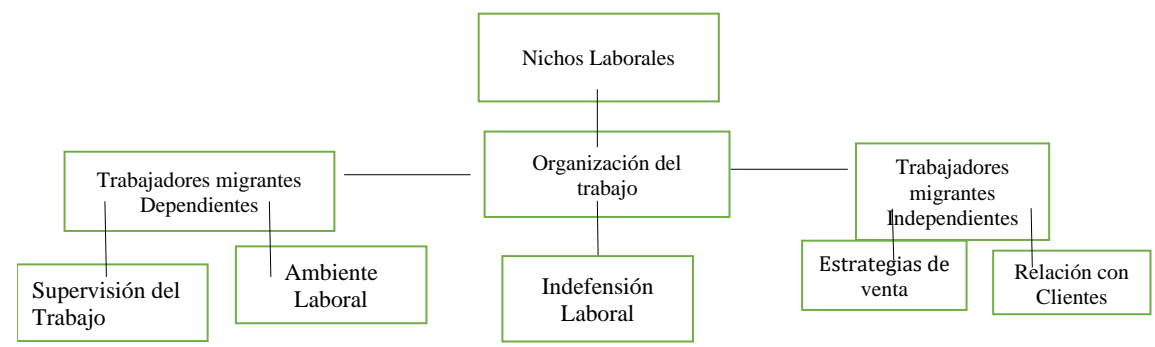

Nichos Laborales, constituye la categoría principal, ya que relaciona tanto a trabajadores dependientes como trabajadores independientes (categorías) y las áreas laborales en que se insertan.

"Mira aquí me tocó asesora de hogar y digo me tocó porque no era lo que venía en mis planes pues, cuando me dijeron vente aquí trabajamos, montamos un negocio o sea era la mentalidad que yo traía, pero por la parte legal me costó el primer lugar de estar acá" (Trabajadora dependiente 1).

"Soy comerciante ambulante... Hay veces que los carabineros no nos molestan tanto, aprovechamos el tiempo, pero cuando no se puede, ya no se puede, porque nosotros arriesgamos lo que nosotros tenemos, eso es lo que nos da de comer a nosotros, eso es lo que nos da el sustento todos los días, así que tratamos de ver" (trabajadora independiente 1).

Organización del trabajo es la primera categoría debido a que constituye la descripción de como trabajadores dependientes e independientes desarrollan sus labores.

“(...) En mi país se trabaja como 8 horas por día y acá yo conozco se trabaja por 9 horas por día ¿sí? Es que cuando yo trabajo 2 horas más o yo trabajar como 4 horas más, se paga a mí la hora extra, eso, yo conozco eso (Trabajador dependiente 1)" . "Hay veces que los carabineros no nos molestan tanto, aprovechamos el tiempo, pero cuando no se puede, ya no se puede, porque nosotros arriesgamos lo que nosotros tenemos, eso es lo que nos da de comer a nosotros, eso es lo que nos da el sustento todos los días, así que tratamos de ver" (Trabajadora Independiente 1).

Supervisión del trabajo se desprende como subcategoría de trabajadores dependientes, ya que son quienes se encuentran bajo las instrucciones de un tercero, da a conocer como es la relación del trabajador migrante y su jefe.

"Excelente, con mi jefa me llevo muy bien" (Trabajadora Dependiente 2).

Ambiente laboral, es otra subcategoría vinculada, pues da a conocer cómo se relaciona con la forma en que el trabajador/a migrante percibe la relación entre él/ella y el resto de los trabajadores.

"Es bueno, en general siempre hay alguien que tiene el carácter más fuerte o no quiere hacer el trabajo, pero el resto el ambiente es tranquilo" (Trabajadora Dependiente 2). 


\section{RTR \\ Revista Territorios y Regionalismos $\mathrm{N}^{\circ} 3,2020$, pp. 82-100 \\ https://doi.org/10.29393/RTR3-6AHML10006}

Estrategias de venta y Relación con Clientes, es una subcategoría de trabajadores independientes, pues se relacionan con la forma de acercarse a sus clientes para vender sus productos y como es el trato entre vendedor y cliente.

"No hay personas groseras en ese sentido, nosotros sabemos que todas las personas merecen respeto, nosotros les tratamos con respeto con cariño, igual ellos las personas igual, bueno a mí me ha tocado así no sé a otras personas" (Trabadora Independiente 1).

Indefensión Laboral es la segunda categoría, pues guarda relación con la carencia y/o falta en el ámbito del trabajo

"Las condiciones en las que nosotros trabajamos, más que todo en el invierno, se nos es difícil trabajar, en el invierno más que todo, ahí es cuando se nos hace más difícil a nosotros, el invierno cuando hay días lluviosos uno no puede ni salir, y yo creo que en eso ya sería que somos vulnerables" (Trabajador Independiente).

El trabajo para los entrevistados representa una oportunidad de inserción no solo laboral, sino también social, ya que les permite conocer la sociedad de destino mediante la interacción con los nacionales, sin embargo, esto muchas veces trae expectativas que tensionan sus prácticas laborales, $y$, por supuesto, su elección y/o continuidad en un empleo determinado, debido a que muchas veces consideran el tener un negocio de forma independiente, ya que representa seguridad laboral y dirigir sus tiempos acorde a lo que ellos esperan.

\section{Impacto en esferas sociales y ampliadas del trabajo}

Se busca visibilizar como el mundo del trabajo incide en las dimensiones socioafectivas del trabajador/a migrante y como esta se ha flexibilizado, considera cinco preguntas.

Figura 3: Resumen de Impacto en esferas sociales y ampliadas del trabajo

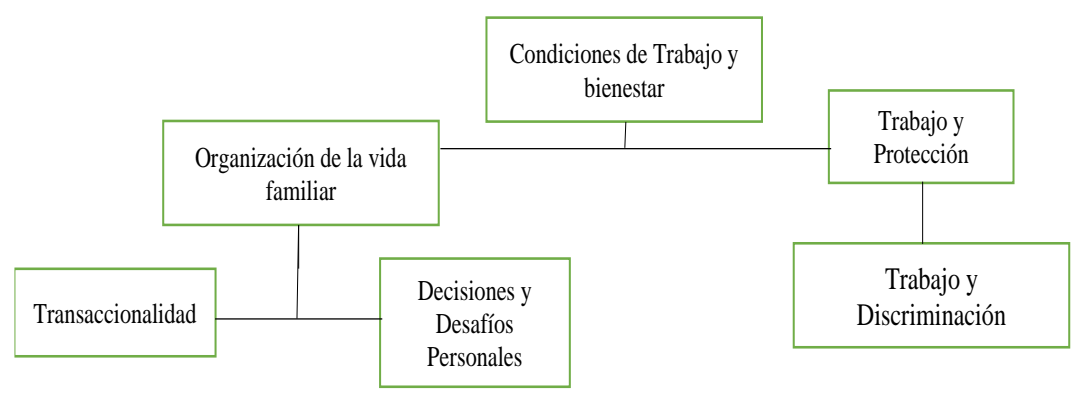

Condiciones de Trabajo y Bienestar constituye la categoría principal, se vincula con los efectos de las condiciones de trabajo en el bienestar individual y familiar 


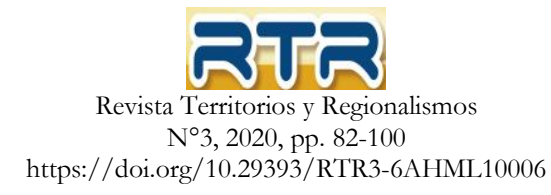

“¿Inseguridad? Hm, yo creo que sí, porque no sabes lo que te puede pasar en el día, lo que te puede pasar y eso, o sea no se sabe que es lo que va a pasar, no se sabe" (Trabajadora Independiente 2).

La primera categoría es Organización de la Vida Familiar referida a la organización de tareas y tiempos del hogar y vida familiar

"se perdió el tiempo para la familia, no sé cómo llamar, esto es que me duele, yo creo que esto he pasado situaciones como que difíciles, complicadas, pero yo creo el que se desvaneció, de que se quebró puedo decir así la familia”.

La primera subcategoría relacionada a este ámbito es la Transnacionalidad, asociada a la relación de los trabajadores/migrantes con su familia en su país de origen, mientras se desenvuelven en el país de destino, relacionada directamente con la segunda subcategoría es Decisiones y desafíos personales, relacionado al lugar que ocupa el trabajo en la adopción de decisiones y desafíos personales

"Poder ejercer mi carrera aquí podría ayudar a traer a mi hermano y colaborar más con mi familia, que es lo más importante, dejaría la cocina y podría hacer lo mío que es lo que me gusta que es mi profesión".

La segunda categoría es Trabajo Protección, relacionando el trabajo y la protección en salud y previsión tanto individual como familiar.

"Fui a pedir una hora, un espacio por Fonasa para un chequeo ginecológico y me dijeron que para noviembre y yo fui en septiembre y yo hombre si fuera una emergencia ... como también me tocó en una oportunidad no tenía el Fonasa... Ahí si extrañaba Venezuela, verdad que ahí si porque yo decía no puede ser posible que no atiendan una emergencia como tal, no sé si es normal acá..." (Trabajadora dependiente 2).

Trabajo y Discriminación como subcategoría se vincula a los efectos de la presencia y/o ausencia de discriminación y desigualdad del trabajo en el sujeto

"(...) Una vez estuvimos ahí, trabajando y una señora se acerca y dice " $¿ Y$ ustedes hasta cuando piensan estar aquí estorbando en la calle?" así nos dice y, pero siempre nos dicen a nosotros no más y no a los chilenos, igual hay chilenos que trabajan ahí entonces solo nos atacan a nosotros porque somos extranjeros, yo creo que por eso y dicen y yo le digo "¿Por qué no le dicen a los chilenos también? Porque no solo somos nosotros si no ellos también y ella vino y me quiso agredir" (Trabajadora Independiente 2).

Las esferas sociales y ampliadas del trabajo son donde las condiciones de precariedad tendrían su mayor incidencia, debido a la situación laboral de los trabajadores/as migrantes dependientes e independientes, ya que las extensas jornadas laborales suponen la pérdida de la vida social y familiar de los migrantes. Por otra parte, se evidencia un fuerte vínculo con el país de origen, pues las trabajadoras migrantes dependientes buscarían la reunificación familiar trayendo consigo a parte de su familia.

\section{Asociatividad, solidaridades y formas de acción colectiva}


La presente dimensión se relaciona con las experiencias de trabajadores/as migrantes y colectivos, organizaciones de migrantes y/o organizaciones sindicales, considera cuatro preguntas.

Figura 4: Resumen de Asociatividad, solidaridades y formas de enfrentar conflictos.

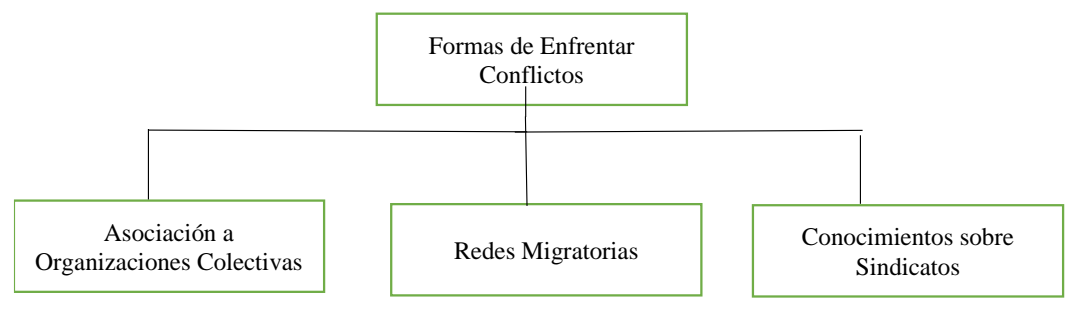

Formas de Enfrentar Conflictos es la categoría principal y se relaciona con describir los principales problemas dentro el contexto de trabajo y formas deseables de resolución de conflictos.

"La verdad conversando y llegar a un acuerdo de buena forma, y que las decisiones las tomemos entre todos, de que lleguemos a un acuerdo de trabajar en grupo y hacer el trabajo por igual" (Trabajador Dependiente 2).

Asociación a Organizaciones Colectivas es la primera categoría, relacionada con la participación de los trabajadores/as migrantes en organizaciones colectivas y/o sindicales.

"Desconozco, te mentiría si digo, ahorita la única institución es Organización X que conocí a F1, es la única institución así, pero del resto... Nada" (Trabajadora Dependiente 1).

La segunda categoría Redes Migratorias, comprende los vínculos entre los entrevistados y otros migrantes, con quienes se apoyan en la inserción laboral en la sociedad de destino

"Eh, si por medio del contacto de mi expareja, a él lo habían llamado para este trabajo, pero como él ya tenía me lo ofreció a mí y fui para allá y bueno allí quedé" (Trabajadora Dependiente 2).

Conocimiento sobre Sindicatos, es la última categoría para esta dimensión y pretende visibilizar y relacionar el mundo sindical con la migración

"Hmm no, tengo un poco de conocimiento de los sindicatos, pero nunca he conocido, he escuchado que hay sindicatos, pero nunca he conocido" (Trabajador Independiente).

\section{Percepción de Precariedad y Realidades del trabajo}

Los entrevistados, dan sus definiciones sobre precariedad, lo cual sugiere nociones básicas que la relacionan con la carencia de condiciones laborales, se consideran tres preguntas. 
Figura 5: Resumen de Percepción de Precariedad y Realidades del trabajo.

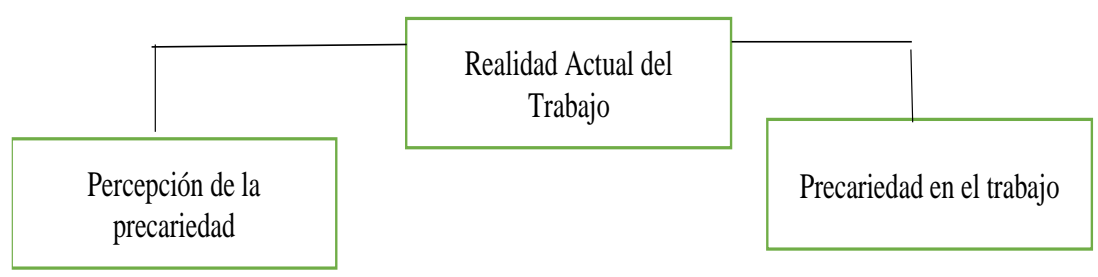

Realidad en el trabajo es la categoría principal los trabajadores/as migrantes entregan su visión sobre la realidad actual del trabajo que realizan, en ellas se evidencia la necesidad de un dialogo que permita establecer nuevas disposiciones que faciliten la inserción del migrante en el país de destino, ya que muchos de ellos son migrantes cualificados.

"Bueno de que allá un poco más de aceptación para todos los inmigrantes como tal, eh y que o sea los que somos profesionales tengamos la oportunidad de poder ejercer, de aportar nuestros conocimientos en alguna parte de mundo del trabajo, sin que involucre tantos procedimientos o tanta burocracia, porque por ejemplo para mí que soy médico... Si ya tú tienes un título y vienes calificado de un país creo que nos deberían de abrir un poquito más las puertas en ese aspecto. Darnos esa oportunidad de que vean que si estamos preparados y estamos bien calificados y a ejercer lo que tantos años estudiamos en Venezuela"

Percepción de Precariedad primera categoría, se relaciona con la definición conceptual que otorgan los trabajadores/as migrantes a este concepto

"Es no tener la seguridad de proveer, yo como padre de familia, no saber cómo pagar el arriendo, el abrigo y por supuesto la comida y las necesidades primarias de la familia, si uno no puede responder a esta" (Trabajador Independiente 1)

Precariedad en el trabajo, segunda categoría que visibiliza la eventual presencia o ausencia de precariedad en el contexto laboral del trabajador/a migrante.

"Como lo dije anteriormente, con un permiso uno tiene un techo y como trabajar o lasas cosas como con que cubrirse para que no se nos moje o se echen a perder las cosas" (Trabajador Independiente 1)

La inserción laboral en la sociedad de destino fue gracias a las organizaciones colectivas y sus contactos, ello sugiere que tales agrupaciones serían claves a la hora de orientar e informar al trabajador/a migrante. Los trabajadores migrantes participantes de esta investigación no tendrían relación con los sindicatos debido a una desvinculación organizacional desde su país de origen, por lo que es de vital importancia conocer y dar a conocer la realidad sindical de la sociedad de destino. 


\section{Caminos de encuentro. Sindicalismo y Migración}

Actividad realizada el día 07 de diciembre de 2018, tuvo como eje central acercar las realidades de migrantes al mundo sindical, se desarrolló con la presencia de dos dirigentes sindicales de la Ciudad y contó con un trabajador de nacionalidad haitiana que se encuentra asociado a un sindicato, para este análisis se consideran apuntes acerca de los principales puntos tratados por cada expositor.

Figura 6: Elaboración Propia.

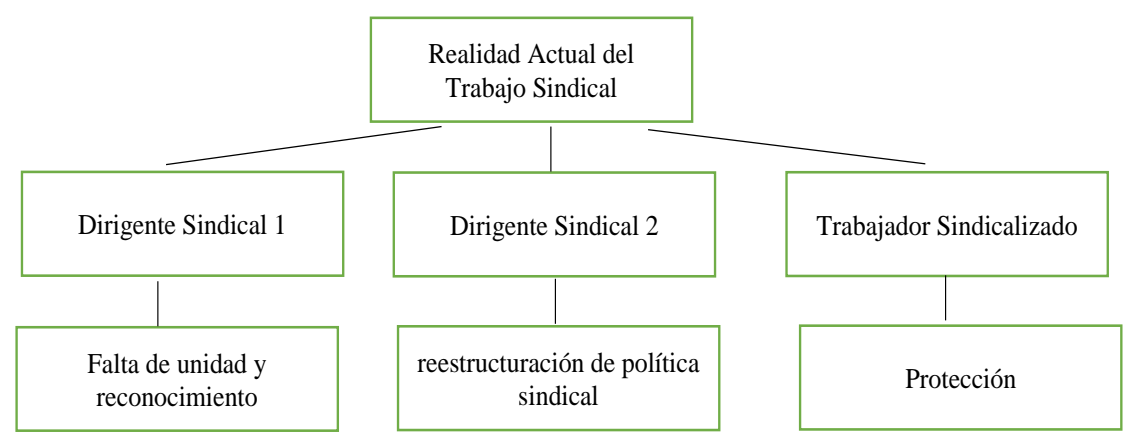

Realidad Actual del Trabajo Sindical, es la categoría principal la cual se desprende del objetivo general de la actividad.

"Generar un espacio de discusión y análisis en torno a la realidad laboral de las y los trabajadores migrantes en la cuidad de Temuco, en conjunto con las organizaciones sindicales, desde sus experiencias vinculadas a la precariedad laboral, distintos tipos de discriminación, y el rol que cumplen los sindicatos de trabajadores tanto para chilenos como migrantes en la defensa de sus derechos laborales" (Propuesta conversatorio p.1).

Falta de unidad y reconocimiento, es la primera categoría se desprende de la exposición del primer expositor/a

"La parte humana también es importante, pues cada persona tiene su propia historia, sus costumbres y su propia cultura, lo cual más que un problema representa un enriquecimiento a todas las culturas que se encuentran presentes en nuestro país, principalmente en nuestro territorio, asimismo es importante reconocer lo positivo de este proceso, pues Chile es un país que está envejeciendo y la llegada de migrantes posibilitarán un aumento en la población y cambiarán nuestra fisonomía, así como nuestra forma de ver el mundo" (Resumen conversatorio p.1).

Reestructuración de política sindical, es la segunda subcategoría, que se desprende de la exposición del segundo/a dirigente sindical.

"Los sindicatos, tienen muchos desafíos por delante, en este sentido la expositora plantea la importancia de mantener una lucha por los derechos de los y las trabajadores migrantes y conaciones, apuntando hacía un reconocimiento de la trayectoria laboral de el o la trabajador (a)... Consideró la necesidad de visibilizar una de las más grandes 


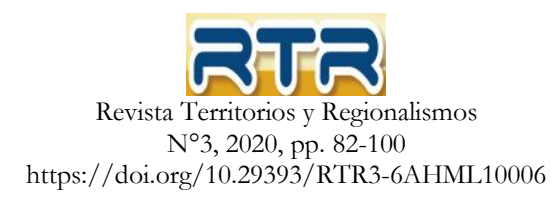

problemáticas de los y las trabajadores como lo son las enfermedades laborales, ya que en muchos casos se deja a la deriva a las familias que se encuentran detrás de ellos... El rol de los sindicatos, particularmente de los dirigentes sindicales fue otro de los puntos centrales de su exposición, ya que apunta hacía dejar de lado la comodidad de los "beneficios" que implica ser un líder sindical" (Resumen conversatorio p.2)

Protección, es la última subcategoría que se desprende de los dichos del trabajador sindicalizado quien señala,

"Las ayudas recibidas de la empresa fueron vitales para poder insertarse en el mundo del trabajo, así como también destaca del compañerismo existente con quienes conforman el sindicato. Sin embargo ... Existe un choque emocional, personal y profesional muy fuerte, dado que en su país ejercía algo completamente distinto al rol que cumple por estos días al interior de la empresa" (Resumen exposiciones conversatorio, p.3).

Los sindicatos deben trabajar de acuerdo con las problemáticas que aquejan a los grupos de trabajadores más vulnerables, entre los que se encuentran los trabajadores migrantes. Este trabajo debe ser de manera mancomunada y debe apuntar hacia construir un dialogo entre los representantes sindicales y las jefaturas en torno a una mayor preocupación por la calidad de vida de los trabajadores, tanto al interior de la empresa como fuera de ella (horarios, alimentación, transporte, descanso).

\section{Discusión}

Las políticas migratorias a nivel regional y global se han configurado en torno a un sistema económico que ha cambiado el mundo del trabajo, empobreciendo a la antigua clase fabril convirtiéndola en empleados de servicios mal remunerados ${ }^{27}$.

La precariedad laboral asociada a los trabajadores migrantes que fueron parte de este estudio, refleja que tanto trabajadores migrantes como nacionales (dependientes o independientes) se encuentran vivenciando inseguridad laboral, ya que el mundo del trabajo a nivel mundial está pasando por una crisis, donde los Estados han reemplazado el enfoque de derecho, el cual ve al trabajador/a como sujeto que se relaciona en múltiples dimensiones, por una mirada economicista centrada en disminuir los costos de producción como señala Grabendorff 28 . Ello representa un retroceso hacia las perspectivas clásicas como la teoría de la dependencia, propuesta por Cardoso y Faletto ${ }^{29}$, ya que se está volviendo a ver al migrante como un medio para conseguir un fin económico (reducción de costos), y no un fin en sí como mismo con motivaciones más allá de lo económico, pasando por alto las dimensiones socioafectivas o elecciones personales.

Las dimensiones de la vida del trabajo no se lograron separar, debido a que para los entrevistados fue de suma importancia considerar a sus familias en su proyecto migratorio y proyecciones a futuro (véase figura 1). Otro motivo para considerar se relaciona con los trabajadores, quienes requieren sus puntos de apoyo que son sus familias y experiencias laborales. Esto contrapone

\footnotetext{
${ }^{27}$ Martínez, op. cit.; Julián, 2014, op. cit:; Carolina Stefoni \& Fernanda Stang, "La construcción del campo de estudio de las migraciones en Chile: notas de un ejercicio reflexivo y autocrítico”, Íconos - Revista de Ciencias Sociales, 0(58), pp. 109-129. https://doi.org/10.17141/iconos.58.2017.2477, 2017; Várguez, op. cit.

28 Wolf Grabendorff, "Los dueños de la globalización: Cómo los actores transnacionales desmantelan el Estado (latinoamericano)", Nueva Sociedad, (271), pp. 55-69, 2017

${ }^{29}$ Cardoso y Faletto, op. cit.
} 


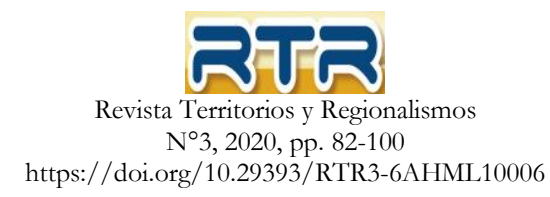

con lo señalado por Waldinger ${ }^{30}$, ya que todos los entrevistados expresaron una añoranza por su país de origen.

Los resultados sugieren que tanto trabajadores hombres como mujeres vivenciarían precariedad laboral, aun cuando no se reconozcan como personas en esa situación, lo cual puede considerarse como un aporte al conocimiento para efectos de la temática y el contexto en el que se inserta esta investigación, ya que de acuerdo, con Tapia ${ }^{31}$, no sólo se considera el género, sino que también su nacionalidad y si tiene o no familia origen, aunque principalmente importa si tiene a su familia en el país de destino. Esto posibilitaría que los trabajadores se inclinen por la elección de empleos que les permitan reducir sus costos de vida, con el propósito de ahorrar y disponer de dinero para fines sociofamiliares, vinculando al migrante a condiciones de inseguridad e inestabilidad asociadas a una precarización del trabajo y a una multidemsionalidad, afectando al trabajador en sus espacios individuales y colectivos ${ }^{32}$.

Los resultados dan cuenta de la importancia de las redes entre migrantes y organizaciones sociales del país de destino, ya que representan una forma de inserción laboral y apoyo psicoemocional frente a situaciones adversas en las múltiples dimensiones en las que se suele hablar de riesgos e inseguridades. Esto solo considerando el tiempo que dure la adaptación en la sociedad de destino, lo cual apoyaría la tesis de Gómez y Torres ${ }^{33}$ donde no existiría mayor relación con dichas organizaciones, debido al poco tiempo que disponen los trabajadores.

El sindicalismo representa para efectos de esta investigación una interrogante para todos los entrevistados/as, dado que se prioriza la búsqueda de trabajo y la preocupación por la socia afectividad comprendida como la manera más importante de mantener una maternidad o paternidad acorde a las necesidades de sus hijos, lo cual motivó su proceso migratorio. Los grupos y organizaciones colectivas de migrantes que se encuentran activos en la Ciudad. Ello sería producto de la flexibilización del trabajo ${ }^{34}$, impidiendo a la persona concebirse capaz de participar activamente y construir una vida social idónea en el país de destino. Frente a ello se considera oportuno lo señalado por Julián ${ }^{35}$, quien plantea la necesidad de establecer diálogos en primer lugar al interior del mundo sindical respecto de las distintas problemáticas vividas como trabajadores para poder visibilizarlas con otros actores del mundo del trabajo, logrando así cambios profundos en la legislación laboral.

La relevancia de esta investigación está marcada por la mirada transnacional de la migración, pues en la actualidad se diversifican las razones y motivaciones por las cuales hombres y mujeres dejan su país de origen, por lo que es necesario adentrarse en su historia de vida para comprender al sujeto social más allá del trabajador migrante. Sin embargo Parella y Cavalcanti ${ }^{36}$ reconocen que la existencia de una situación socio económica en su país de origen los condicionó a tomar la decisión de emigrar, sacrificando su bienestar familiar, con el propósito de buscar una mejor valoración sobre su actividad laboral en otro país, que les permita ejercer de mejor forma la paternidad o maternidad respectivamente a nivel económico pues se visibiliza una preocupación por darles una buena educación y un buen cuidado a sus hijos por encima de las comodidades o las implicancias prácticas de lo que significa migrar.

Las prácticas sociales en relación a las migraciones llaman a empatizar acerca de las condiciones en las que padres y madres dejan de lado sus distintos roles en una sociedad para velar por los interésese de sus hijos, lo cual muchas veces implica dejar sus propias historias de logro y

\footnotetext{
${ }^{30}$ Waldinger, op. cit.

31 Tapia, op. cit.

32 Julián, 2014, op. cit.

33 Gómez y Torres, op. cit.

34 Martínez, op. cit:; Julián, 2016, op. cit:; Julián, 2017, op. cit.

35 Julián, 2014, op. cit:; Julián, 2016, op. cit.

36 Parella \& Cavalcanti, op. cit.
} 


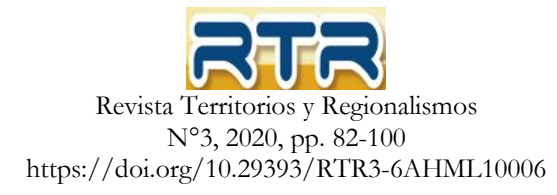

comodidad con el propósito de conseguir con esfuerzo mejores y mayores oportunidades para los miembros de su familia, quitando el factor económico, esto se relacionaría con una estabilidad social propia del país de destino. Se propone en base a este trabajo una mirada más comprensiva e integrada en el mundo del trabajo, donde converjan lo económico con las relaciones socio familiares y afectivas de los trabajadores, ya que parte fundamental de un trabajador saludable es contar con una temporalidad acorde que le permita desenvolverse en todas las esferas sociales de las cuales forma parte, siendo la más importante de ellas la dimensión socioafectiva, Zapata ${ }^{37}$ (2016), plantea que para lo cual se necesitan profesionales y políticas más humanizadoras en el mundo del trabajo que permitan prestar ayuda oportuna y pertinente a cada situación particular de cada trabajador, independientemente si es migrante o nacional, pues de lo contrario se estaría discriminando a los trabajadores/as, aumentando aún más la desvalorización existente de la fuerza del trabajo, provocando así una disminución de las economías mundiales, trayendo consigo crisis sociopolíticas, hambrunas y escasez.

Futuras investigaciones podrían profundizar acerca del auto reconocimiento del migrante como sujeto de derechos y su vinculación en dos ámbitos laborales: trabajadores dependientes o dependientes, ya que de acuerdo con la pequeña muestra considerada en esta investigación, se constata la indefensión en la que se encontrarían los trabajadores independientes sobre todo en materia de salud y previsión social, mientras que los trabajadores dependientes, si bien están más resguardados en ese aspecto, estarían más propensos a sufrir sobre explotación ante la necesidad de conseguir dinero para cumplir con sus metas y desafíos personales asociados a la transaccionalidad ${ }^{38}$.

Las oportunidades y herramientas de crear una mejor sociedad las tienen aquellas personas que ven más allá de sí mismas y velen por aquellos que desconocen a un otro, con mismas características, sueños, anhelos y metas por cumplir, que simplemente no han tenido las mismas oportunidades, pero que aun así buscan alguien que los guie y acompañé en la búsqueda de nuevas oportunidades que les permitan un desarrollo y crecimiento, personal, familiar y laboral, solo se debe ir más allá de lo aparente.

\section{Bibliografía}

ÁVILA JAVIER, "Repensando la etnicidad y el transnacionalismo desde el análisis de redes personales", Redes. Revista hispana para el análisis de redes sociales, 26(2), pp. 158-170, 2015

BORDA FALS, Una sociología sentipensante para América Latina, (No. 316 301.098). e-libro, Corp, 2009

CARDOSO FERNANDO \& ENZO FALETTO, Dependencia y desarrollo en América Latina: ensayo de interpretación sociologica, (No. 330.13/C26d), Siglo Veintiuno, 1971

CHÁVEZ GUILLERMINA \& SUSANA PRECIADOS, "Comunidad transnacional e interacción comunicativa: diagnóstico social" PROSPECTIVA. Revista de Trabajo Social e Intervención Social, pp.37-62, 2018

COMISIÓN NACIONAL DE INVESTIGACIÓN CIENTÍFICA Y TECNOLÓGICA, "Declaración de Singapur sobre la integridad en la investigación", link de consulta: https://www.conicyt.cl/fondap/files/2014/12/DECLARACI\%C3\%93NSINGAPUR.pdf

CORRAL YADIRA, "Validez y Fiabilidad en Investigaciones Cualitativas", Revista Arjé, pp. 11-14, 2017

GÓMEZ PABLO \& VÍCTOR TORRES, "Redes sociales en la migración paraguaya y brasilera hacia la Argentina. Un análisis comparativo de las redes de ayuda y los vínculos binacionales", Anais, pp. 1-20, 2016

\footnotetext{
37 Zapata, op. cit.

38 Parella \& Cavalcanti, op. cit.
} 


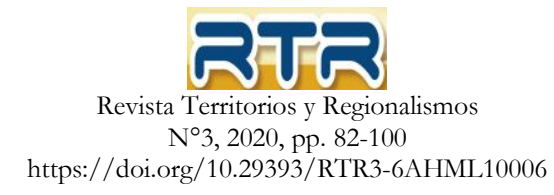

GRABENDORFF WOLF, "Los dueños de la globalización: Cómo los actores transnacionales desmantelan el Estado (latinoamericano)", Nueva Sociedad, (271), pp. 55-69, 2017

HERNÁNDEZ ROBERTO, CARLOS FERNÁNDEZ \& PILAR BAPTISTA, Metodología de la investigación (5a ed.), McGraw-Hill, México, D.F, 2010

JULIÁN DASTEN, "Identidades y resignificados del actor sindical. Diez Núcleos de tensión del sindicalismo en Chile", Andamios, 13(30), pp. 171-194, 2016

JULIÁN DASTEN, "La precariedad laboral, modernidad y modernización capitalista: Una contribución al debate desde América Latina", Trabajo y sociedad, (23), pp. 147-168, 2014

JULIAN DASTEN, "Precariedad laboral en América Latina: contribuciones a un modelo pata armar", Revista Colombiana de Sociología, 40(2), pp. 27-46, 2017

MABOGUNJE AKIN, "Systems Approach to a Theory of Rural-Urban Migration", Geographical Analysis, v. 2, n. 1, p. 1-18, 1970

MARTÍNEZ ARTURO, "Estrategia Sindical en un Chile Internacionalizado", en Jaime Ensignia (editor), Mitos y realidades del mercado laboral en Chile, pp.199-212, Friedrich Ebert Stiftung, Santiago, 2005

MORET JOELLE, "Cross-border mobility, transnationality and ethnicity as resources: european Somalis' post-migration mobility practices", Journal of Ethnic and Migration Studies, 42(9), pp. 1455-1472, 2016

PARELLA SONIA \& LEONARDO CAVALCANTI, "Aplicación de los campos sociales transnacionales en los estudios sobre migraciones", en C. Solé, S. Parella, L. Cavalcanti (Coords.), Nuevos retos del transnacionalismo en el estudio de las migraciones, Ministerio de Trabajo e Inmigración, Subdirección General de Información Administrativa y Publicaciones, Madrid, 2009

PARELLA SONIA, Mujer, inmigrante y trabajadora: La triple discriminación, Anthropos Editorial, Barcelona, 2003

PINILLA FRANCISCO, La degradación de las condiciones de trabajo: Tendencias de flexibilización en las relaciones de empleo, intensificación del esfuerzo y consecuencias en la salud de los trabajadores, Doctoral dissertation, Universidad Nacional de Educación a Distancia., UNED, 2015

RAVENSTEIN ERNST, "The laws of migration", Journal of the statistical society of London, 48(2), pp. 167235,1885

STEFONI CAROLINA \& FERNANDA STANG, "La construcción del campo de estudio de las migraciones en Chile: notas de un ejercicio reflexivo y autocrítico", Íconos - Revista de Ciencias Sociales, 0(58), pp. 109-129. https://doi.org/10.17141/iconos.58.2017.2477, 2017

STEFONI CAROLINA, SANDRA LEIVA \& MACARENA BONHOMME, "Migración internacional y precariedad laboral. El caso de la industria de la construcción en Chile", Revista Interdisciplinar da Mobilidade Humana, 25(49). Recuperado de http://www.csem.org.br/remhu/index.php/remhu/article/view/717,2017

TAPIA LADINO MARCELA, "Frontera, movilidad y circulación reciente de peruanos y bolivianos en el norte de chile", Estudios atacameños, (50), pp. 195-213. https://doi.org/10.4067/S071810432015000100010,2015

TAYLOR STEVEN \& ROBERT BOGDAN, Introducción a los métodos cualitativos de investigación, Paidós, Barcelona, 1990

VÁRGUEZ LUIS, "Francisco Zapata, El sindicalismo latinoamericano”, Estudios sociológicos, 35(104), pp. $457-461,2017$

WALDINGER ROGER, "Más allá del transnacionalismo: Una perspectiva alternativa de la conexión de los inmigrantes con su país de origen", Migraciones Internacionales, 7(1), pp. 189-220. https://doi.org/10.17428/rmi.v7i1.690, 2017 
Revista Territorios y Regionalismos $\mathrm{N}^{\circ} 3,2020$, pp. 82-100

https://doi.org/10.29393/RTR3-6AHML10006

ZAMORANO ALFONSO, "Análisis lógico y análisis gramatical en Colombia (s. XX): la teoría lingüística de Próspero González Ruiz”, Revista argentina de historiografía lingüistica, 2(2), pp. 125-143, 2016

ZAPATA ADRIANA, "Madres y padres en contextos transnacionales: el cuidado desde el género y la familia", Desacatos, (52), pp. 14-31, 2016 